\title{
SiGe Nanowires Grown by LPCVD using Ga-Au Catalysts
}

\author{
M. Monasterio ${ }^{1}$, A. Rodríguez ${ }^{1}$, T. Rodríguez ${ }^{1}$, C. Ballesteros ${ }^{2}$ \\ ${ }^{1}$ Tecnología Electrónica, Universidad Politécnica de Madrid, E.T.S.I.T., 28040 Madrid, Spain \\ ${ }^{2}$ Física, Universidad Carlos III, 28911 Leganés (Madrid), Spain
}

\begin{abstract}
The use of Ga-Au alloys as metal catalysts for the growth of SiGe nanowires has been investigated. The grown nanowires are cylindrical and straight, with a defect-free crystalline structure, sharp nanowire-droplet interfaces and an almost constant Ge atomic fraction throughout all their length. These features represent significant improvements over the results obtained using pure $\mathrm{Au}$.
\end{abstract}

\section{INTRODUCTION}

Group IV ( $\mathrm{Si}, \mathrm{Ge}$ or $\mathrm{SiGe}$ ) nanowires (NWs) have been extensively analyzed because of their potential applications in electronics, optoelectronics, photovoltaics and sensing, as well as due to their compatibility with current device fabrication technologies [1, 2]. The most commonly used catalyst metal for the growth of semiconductor nanowires by the Vapour-LiquidSolid (VLS) method is Au. However, among other drawbacks, the interfaces in Si-SiGe NWs heterostructures show a compositional interfacial broadening of the order of the NW diameter [3]. The VLS growth of Si NWs using other metals, like Ga [4, 5] and In [6], has been considered. These metals show very low solubilities of $\mathrm{Si}$ and $\mathrm{Ge}$, an effect that could be advantageous to improve the abruptness of interfaces in heterostructures. However, they are not catalytic, so conventional chemical vapor deposition (CVD) techniques cannot be used and plasma assisted CVD techniques are required. The use of alloys of catalytic and non-catalytic metals, like $\mathrm{Ga}-\mathrm{Au}$, appears as an alternative to take advantage of the properties of both species using a conventional CVD process. Ga-Au alloys have been used for the growth of Si NWs on $\mathrm{Si}$ by CVD [7]. In this work, the growth of SiGe NWs by CVD using Ga-Au alloys of different compositions as catalysts is investigated. The morphological, structural and compositional properties of the grown NWs are compared with those of the NWs grown using Au.

\section{EXPERIMENTAL}

SiGe NWs of different compositions were grown by the VLS method using a Low Pressure CVD reactor. Bare (100) and (111) as well as thermally oxidized Si wafers were used as substrates and metal films of $\mathrm{Ga}$ and $\mathrm{Au}$ ( $\mathrm{Ga}$ evaporated first) were deposited on top of them by evaporation. The $\mathrm{Ga}$ content was varied from 0 (pure $\mathrm{Au}$ ) to $100 \%$ (pure $\mathrm{Ga}$ ) by changing the expected thickness to be achieved from each evaporation process keeping a constant total thickness of around $10 \mathrm{~nm}$. The intermediate values of the nominal Ga content were $50 \%, 75 \%$ and $90 \%$. Prior to NW growth, the samples were annealed at $500{ }^{\circ} \mathrm{C}$ in hydrogen atmosphere 
inside the reactor. During the subsequent growth process, $\mathrm{Si}_{2} \mathrm{H}_{6}$ and $\mathrm{GeH}_{4}$ were used as precursor gases for $\mathrm{Si}$ and Ge with a $\mathrm{GeH}_{4}: \mathrm{Si}_{2} \mathrm{H}_{6}$ flow ratio (R) varying from 0 to 2 . The total pressure was kept constant at 400 mTorr, the temperature was varied from 400 to $470^{\circ} \mathrm{C}$ depending on the value of $\mathrm{R}$ and the process times ranged from 1 to $15 \mathrm{~min}$.

The as-deposited and annealed substrates as well as the length, diameter and morphology of the grown SiGe NWs were analyzed by Scanning Electron Microscopy (SEM). The crystalline structure and defects of the NWs was studied by Transmission Electron Microscopy (TEM) using NWs separated from the substrate by sonication of the samples and collected in a methanol bath. Diffraction pattern simulation from Fourier transforms (FTP) of the high resolution images was used for these purposes. Compositional studies along the NWs bodies, including the metal droplets, were carried out by an EDX system attached to the TEM.

\section{RESULTS}

\section{Morphological features of the NWs}

The analysis by SEM of the Au and Ga-Au as-deposited metal layers indicates that the surface of the samples is covered by droplets. Their diameter ranges from 10 to $50 \mathrm{~nm}$ in the samples with $50 \% \mathrm{Ga}$, with an average value of $40 \mathrm{~nm}$. The average diameter increases slightly as the Ga content increases, reaching $45 \mathrm{~nm}$ in the samples with $90 \% \mathrm{Ga}$. The annealing step carried out before the NWs growth does not change significantly the distributions of diameters.

A selection of SEM micrographs of NWs is displayed in Figure 1. With regard to the density of NWs, the most significant results can be summarized as follows: a) In the samples with pure $\mathrm{Au}$, densities from $5 \times 10^{6} \mathrm{~cm}^{-2}$ to $6 \times 10^{8} \mathrm{~cm}^{-2}$ in (111) and (100) Si substrates were measured. b) The samples with Ga-Au show NWs densities from $10^{6} \mathrm{~cm}^{-2}$ to $2 \times 10^{8} \mathrm{~cm}^{-2}$ in (111) Si and below $10^{6} \mathrm{~cm}^{-2}$ in (100) Si. It increases progressively as the Ga content in the metal does (at least up to $90 \% \mathrm{Ga}$ ). c) The density of NWs is significantly higher in the oxidized Si samples than in the bare $\mathrm{Si}$ ones for the same growth conditions. d) No growth at all was achieved in samples with pure $\mathrm{Ga}$, showing that the presence of Au is necessary for the growth to occur. An explanation for these results could be based on two opposite phenomena that compete with each other. On one hand, the density of NWs decreases as the amount of Au, the catalytic element responsible for the decomposition of the precursor gases, decreases. On the other hand, the nucleation of NWs is favoured as the amount of Ga increases due to the low solubility of Si and Ge in Ga.

The NWs grown using pure Au show average lengths from 1 to $3 \mu \mathrm{m}$ and average diameters from 20 to $25 \mathrm{~nm}$ depending on the growth conditions. Both magnitudes increase as R decreases and the growth temperature increases. Most of the NWs are curved and tangled, and a mixture of cylindrical and tapered NWs is observed. The density of tapered NWs increases as the flow ratio is increased and they become dominant for $\mathrm{R}>1$.

On the other hand, the NWs grown using Ga-Au are cylindrical and straight and only a small amount of tapered NWs appears for $\mathrm{R}=2$. The average diameters of the NWs grown from $\mathrm{Ga}-\mathrm{Au}$ in different conditions are slightly larger, ranging from 30 to $40 \mathrm{~nm}$ and similar to the diameters of the original droplets. The average lengths are also larger, from 2 to $6 \mu \mathrm{m}$. Again, both magnitudes increase as $\mathrm{R}$ decreases and the growth temperature increases. The dispersion observed in the values of the diameter is around $\pm 5 \mathrm{~nm}$ in all cases, while in the length it decreases from $\pm 3 \mu \mathrm{m}$ (average value: $6 \mu \mathrm{m}$ ) for $\mathrm{R}=0$ to $\pm 1 \mu \mathrm{m}$ (average value: $2 \mu \mathrm{m}$ ) for $\mathrm{R}=2$. 
(a) $\mathrm{SiO}_{2}, 75 \% \mathrm{Ga}, \mathrm{R}=0.5,445^{\circ} \mathrm{C}$

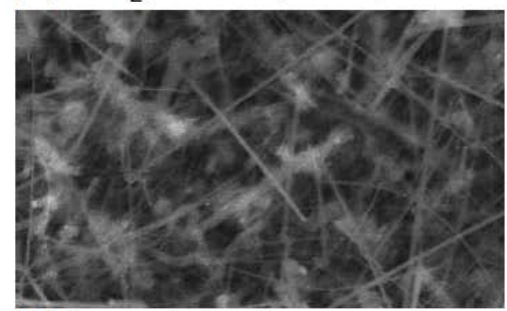

(d) (100) $\mathrm{Si}, \mathrm{Au}, \mathrm{R}=1,5,410^{\circ} \mathrm{C}$

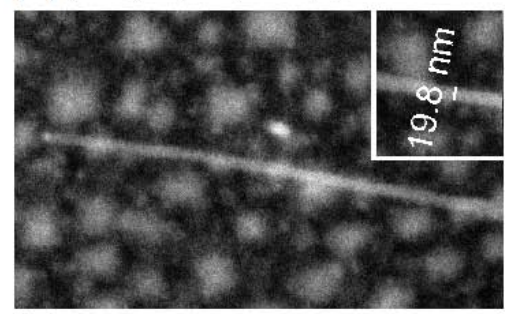

(g) (100) $\mathrm{Si}, 75 \% \mathrm{Ga}, \mathrm{R}=1,430^{\circ} \mathrm{C}$

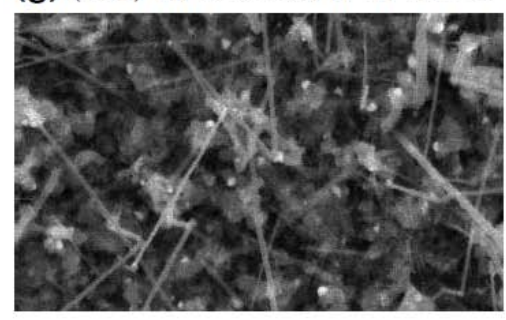

(b) $\mathrm{SiO}_{2}, 50 \% \mathrm{Ga}, \mathrm{R}=1,430^{\circ} \mathrm{C}$

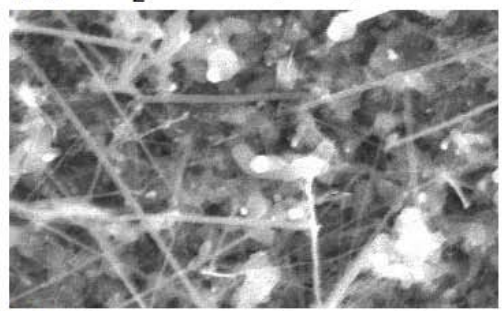

(e) $\mathrm{SiO}_{2}, 75 \% \mathrm{Ga}, \mathrm{R}=1,430^{\circ} \mathrm{C}$

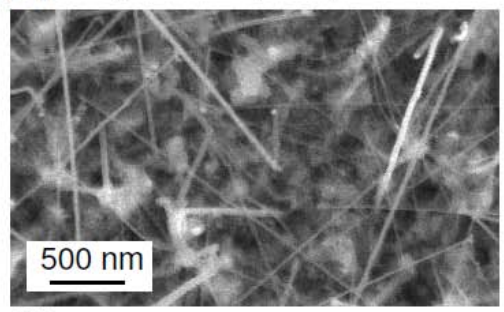

(h) $\mathrm{SiO}_{2}, 90 \% \mathrm{Ga}, \mathrm{R}=1,430^{\circ} \mathrm{C}$

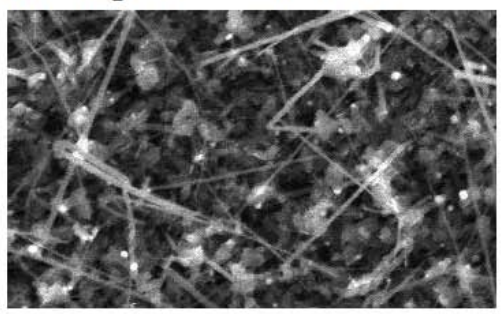

(c) (111) $\mathrm{Si}, 75 \% \mathrm{Ga}, \mathrm{R}=1,430^{\circ} \mathrm{C}$

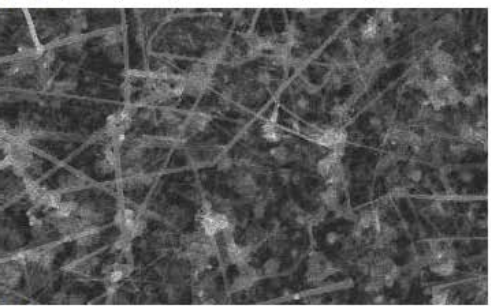

(f) (100) Si, 50\% Ga, R=1,5, $410^{\circ} \mathrm{C}$

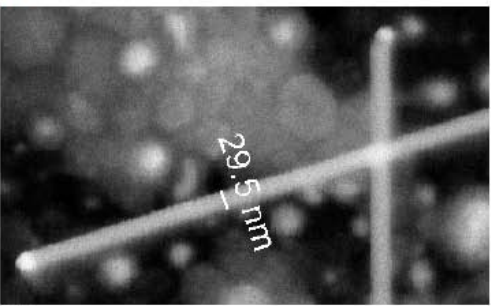

(i) $\mathrm{SiO}_{2}, 75 \% \mathrm{Ga}, \mathrm{R}=2,410^{\circ} \mathrm{C}$

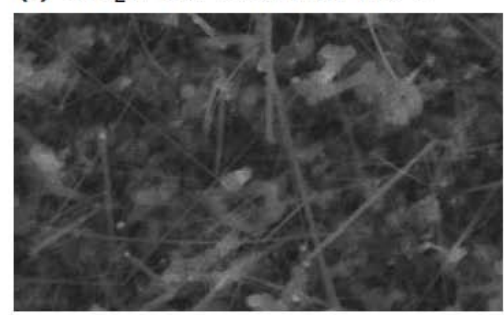

Figure 1. SEM images of NWs grown for $5 \mathrm{~min}$. using different process conditions. Taking the central micrograph (e) of the $3 \times 3$ matrix as a reference, the central column (b, e, h) shows the effect of the Ga content in the metallization, the main diagonal (a, e, i) illustrates the influence of the gas flow ratio and the counterdiagonal (c, e, g) shows the results obtained using different substrates. The central row shows details of a tapered NW grown using Au (d) and a cylindrical NW grown using Ga-Au (f). All images have been obtained with a magnification of $10000 \times$ (see scale bar in the center) except micrographs (d) and (f).

The length of the NWs increases with the process time for growth times up to $5 \mathrm{~min}$. SEM observations show the droplet at the end of the straight NW body and it seems that the NW length is determined by the process time. The growth rates, estimated as the NW length divided by the process time, range from 0.2 to $0.5 \mu \mathrm{m} / \mathrm{min}$. in the NWs grown using pure Au and from 0.4 to $1.2 \mu \mathrm{m} / \mathrm{min}$. in the NWs grown using $\mathrm{Ga}-\mathrm{Au}$. For process times longer than $5 \mathrm{~min}$., however, the NWs length does not increase further. The SEM and TEM images (see later) indicate that after a certain process time, the growth direction changes and the growth is stopped.

\section{Crystalline structure and defects}

Figure 2 (a) shows TEM images of SiGe NWs grown on (100) Si from Au. The droplets located at the end of the NWs exhibit different shapes, they are not well oriented with respect to the NW main axis and the droplet-NW interface is not flat. Most parts of the NWs bodies show amorphous regions and defects like stacking faults, with changes in the crystalline orientation along the NW. The distances and angles measured in the FTP correspond to a diamond structure with structural distortions that could be associated to lattice parameter variations due to 
compositional fluctuations. The outer surface is rough at the atomic scale used and is covered by an oxide layer. The growth of more than one NW from a single Au droplet is usual.

Images of a NW grown on (111) Si using Ga-Au (50\% Ga) are shown in Figure 2 (b). If the process is stopped before the growth is self limited, the droplets are spherical and centered at the end of the NW body. The droplet-NW interface follows the lattice planes of the structure and in most cases is sharp, flat within a few atomic layers and perpendicular to the NW axis. The body of the NWs is monocrystalline and defect-free. The main axis of the NWs is oriented along the $\langle 111\rangle$ direction and the crystalline structure is diamond-like, with a slight distortion in some cases. The outer surface is smooth at the atomic scale used and is also covered by oxide. The same features are observed in the NWs grown on $\mathrm{SiO}_{2}$ using Ga-Au. In the NWs which exhibit changes in the growth direction prior to the self-stopping of the growth, Figure 2 (c), Wurtzite structure appears in the region next to the droplet, where no Ge is detected in the NW (see later).

(a) (100) $\mathrm{Si}, \mathrm{Au}, \mathrm{R}=1,430 \stackrel{\circ}{\mathrm{C}}$
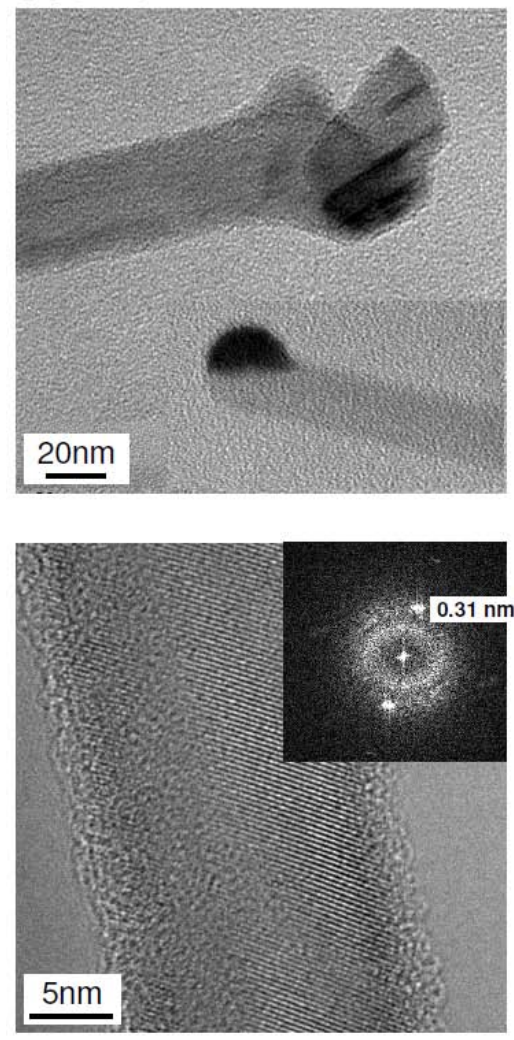

(b) (111) $\mathrm{Si}, 50 \% \mathrm{Ga}, \mathrm{R}=1,430 \stackrel{\circ}{\mathrm{C}}$
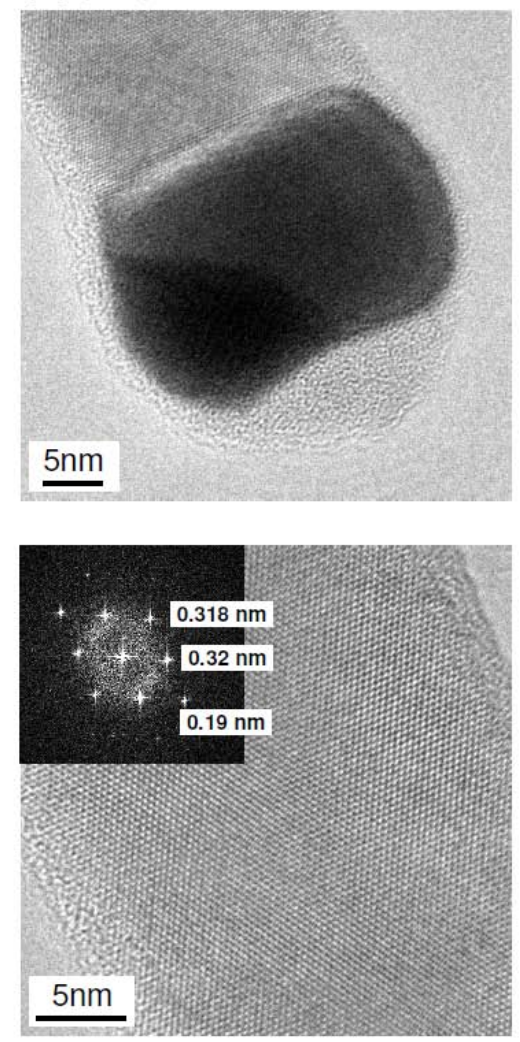

(c) $\mathrm{SiO}_{2}, 50 \% \mathrm{Ga}, \mathrm{R}=1,430 \stackrel{\circ}{ } \mathrm{C}$
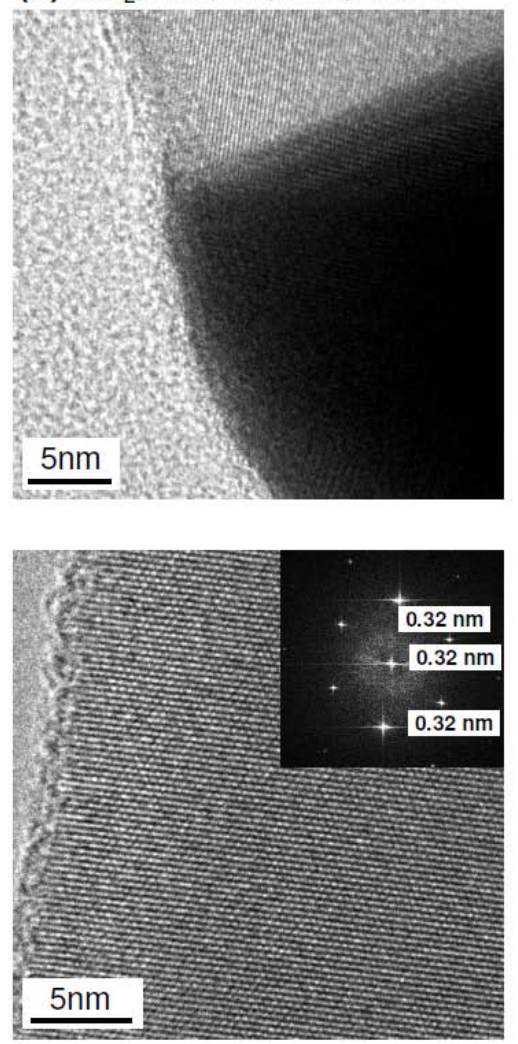

Figure 2. TEM images of NWs grown at $430^{\circ} \mathrm{C}$ with $\mathrm{R}=1$ using (a) $\mathrm{Au}$, (b) Ga-Au (50\% Ga) on (111) Si and (c) $\mathrm{Ga}-\mathrm{Au}(50 \% \mathrm{Ga})$ on Si oxide. Upper part: droplet and its interface with the NW body. Lower part: high resolution image of the body with its corresponding FTP.

\section{Compositional analysis}

Figure 3 shows some images of individual NWs and the results of the compositional studies carried out by EDX along the bodies of several NWs grown on $\mathrm{SiO}_{2}$. 

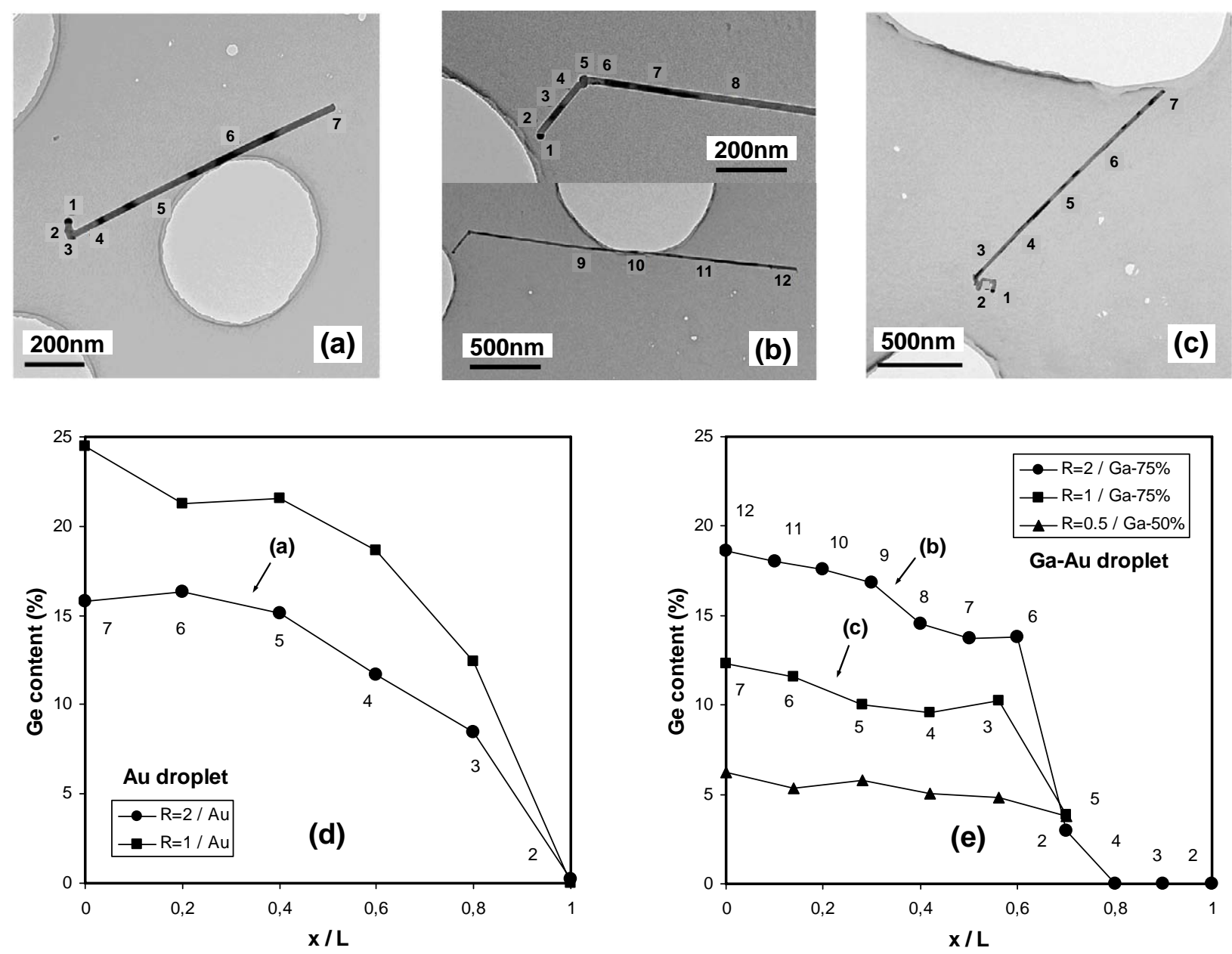

Figure 3. TEM images of NWs grown on $\mathrm{SiO}_{2}$ with (a) $\mathrm{R}=2$, pure $\mathrm{Au}, 410^{\circ} \mathrm{C}$, (b) $\mathrm{R}=2$, Ga- $\mathrm{Au}(75 \% \mathrm{Ga}$ ), $410^{\circ} \mathrm{C}$ and (c) $\mathrm{R}=1$, Ga-Au ( $\left.75 \% \mathrm{Ga}\right), 430^{\circ} \mathrm{C}$. The approximate locations selected for the compositional analysis by EDX are indicated in each case (number 1 corresponds to the droplet). The graphs show the evolution of the Ge content along the NW length in NWs grown from (d) Au and (e) Ga-Au, including the three cases shown in the images displayed above (see labels) and other results.

When the NWs are grown from pure $\mathrm{Au}$, the Ge fraction decreases progressively from a certain value measured at the root of the NW to full absence of Ge in the region located next to the Au droplet. On the other hand, the Ge fraction is nearly constant with the resolution achieved along the whole length in the NWs grown from Ga-Au regardless the Ga content in the original film. As in the previous case, no Ge is detected right next to the droplet located at the end of the NW when the growth direction has changed and the NW growth is self-stopped, but Ge depletion takes place abruptly, instead of progressively. The NW growth stops once Ge depletion takes place, since the temperature selected for the growth of SiGe NWs of a certain composition is too low for the noticeable growth of Si NWs. This is the region where Wurtzite structure is observed.

Figure 4 shows that the Ge atomic content in the bodies of the NWs grown using Ga-Au increases almost linearly with the gas flow ratio up to a value of around $20 \%$ for $\mathrm{R}=2$. The linear dependence holds for the different values of the Ga content in the original film, and the measured values of the Ge atomic fraction are similar for a given Ga content within the uncertainity in the results, so the decomposition of the reactants by the catalytic action of Au seems to be the factor 
that determines the Ge content in the NWs. The analysis of the droplets located at the end of the NWs shows that they contain Ga, although usually less than the original Ga-Au films, so it is clear that $\mathrm{Ga}$ has played a key role in the growth process.

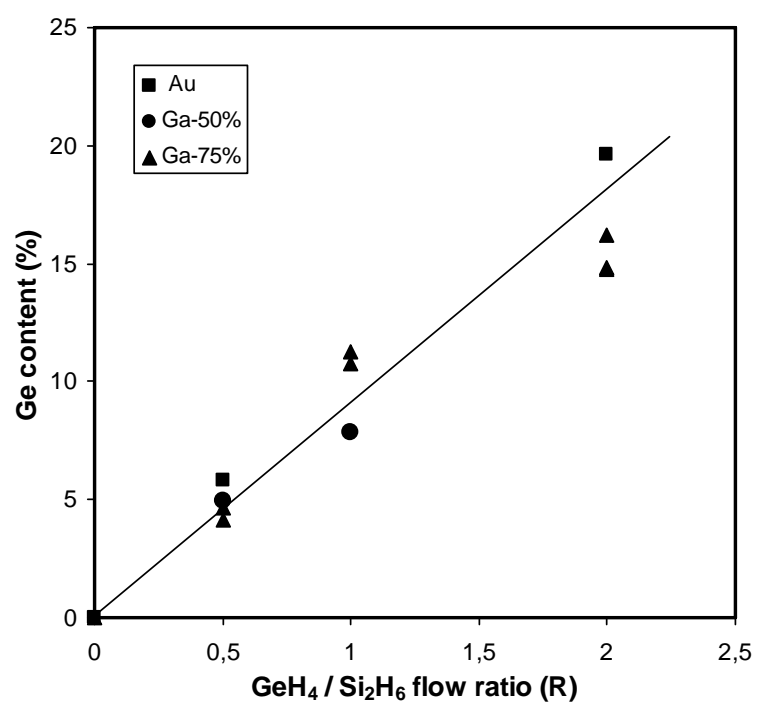

Figure 4. Ge atomic fraction of the NWs as a function of the gas flow ratio and for different metal layers.

\section{CONCLUSIONS}

SiGe NWs of different compositions have been grown by the VLS method using Ga-Au metal films. a) The NWs are mainly cylindrical and straight throughout the whole intervals of growth temperatures $\left(400-470{ }^{\circ} \mathrm{C}\right)$ and $\mathrm{GeH}_{4}: \mathrm{Si}_{2} \mathrm{H}_{6}$ gas flow ratios (0 - 2) considered. b) The bodies of the NWs are monocrystalline and defect-free. c) The Ge content in the NWs varies proportionally to the gas flow ratio and it is almost constant from the root to the droplet located at the end. d) The droplet-NW body interfaces are flat and sharp. These features are advantageous over those of the NWs grown using $\mathrm{Au}$, where tapered NWs with amorphous regions and stacking faults, a non-uniform composition and not well defined interfaces with their droplets are dominant.

\section{ACKNOWLEDGMENTS}

This work was funded by the Spanish Government (Grant: MAT2010-20441-C02-02). TEM work has been carried out at the LABMET of the CAM, Madrid.

\section{REFERENCES}

1. Y. Cui, Q. Wei, C. M. Lieber. Science 293, 1289 (2001).

2. X. Zhang, K. K. Lew, P. Nimmatori, J. Redwing, E.C. Dickey; Nano Lett. 7, 3241 (2007).

3. T. E. Clark, P. Nimmatoori, K. K. Lew, L. Pan, J. M. Redwing, E. C. Dickey. Nano Lett. 8, 1246 (2008).

4. M. K. Sunkara, S. Sharma, R. Miranda, G. Lian, and E. C. Dickey. Appl. Phys. Lett. 79, 1546 (2001).

5. M. Jeon, Y. Tomitsuka and K. Kamisako. Journal of Industrial and Engineering Chemistry 14, 836 (2008).

6. F. Iacopi, P. M. Vereecken, M. Schaekers, M. Caymax, N. Moelans, B. Blanpain, O. Richard, C. Detavernier and H. Griffiths. Nanotechnology 18, 505307 (2007).

7. A. Lugstein, M. Steinmair, Y. J. Hyun, E. Bertagnolli, P. Pongratz. Appl. Phys. Lett 90, 023109 (2007). 